\title{
Author Correction: Concise \\ N-doped Carbon Nanosheets/ Vanadium Nitride Nanoparticles Materials via Intercalative Polymerization for Supercapacitors
}

\author{
Yongtao Tan ${ }^{1,2}$, Ying Liư ${ }^{1,2}$, Zhenghua Tang ${ }^{4,5}$, Zhe Wang ${ }^{6}$, Lingbin Kong ${ }^{1,2}$, Long Kang ${ }^{1,2}$, \\ Zhen Liu L $^{3}$ Fen Ran (iD ${ }^{1,2}$
}

Correction to: Scientific Reports https://doi.org/10.1038/s41598-018-21082-w, published online 13 February 2018

The Acknowledgements section in this Article is incomplete:

“This work was partly supported by the National Natural Science Foundation of China (51203071, 51363014, 51463012, and 51763014), China Postdoctoral Science Foundation (2014M552509, and 2015T81064), Natural Science Funds of the Gansu Province (1506RJZA098), and the Program for Hongliu Distinguished Young Scholars in Lanzhou University of Technology (J201402)."

should read:

“This work was partly supported by the National Natural Science Foundation of China (51203071, 51363014, 51463012, and 51763014), China Postdoctoral Science Foundation (2014M552509, and 2015T81064), Natural Science Funds of the Gansu Province (1506RJZA098), and the Program for Hongliu Distinguished Young Scholars in Lanzhou University of Technology (J201402). Dr. Zhe Wang would like to acknowledge the support from the National Science Foundation (Grant 1700429) and NIMHD-RCMI (5G12MD007595) and the NIGMS-BUILD (8UL1GM118967) and this publication was also made possible by the Louisiana Cancer Research Consortium.”

\footnotetext{
${ }^{1}$ State Key Laboratory of Advanced Processing and Recycling of Non-ferrous Metals, Lanzhou University of Technology, Lanzhou, 730050, P. R. China. ${ }^{2}$ School of Material Science and Engineering, Lanzhou University of Technology, Lanzhou, 730050, Gansu, P. R. China. ${ }^{3}$ Department of Physics \& Engineering, Frostburg State University, Frostburg, MD, 21532-2303, USA. ${ }^{4}$ Guangzhou Key Laboratory for Surface Chemistry of Energy Materials, New Energy Research Institute, School of Environment and Energy, South China University of Technology, Guangzhou Higher Education Mega Centre, Guangzhou, 510006, China. ${ }^{5}$ Guangdong Provincial Key Laboratory of Atmospheric Environment and Pollution Control, Guangdong Provincial Engineering and Technology Research Center for Environmental Risk Prevention and Emergency Disposal, South China University of Technology, Guangzhou Higher Education Mega Centre, Guangzhou, 510006, China. ${ }^{6}$ Department of Chemistry, Xavier University of Louisiana, New Orleans, LA, 70125, USA. Correspondence and requests for materials should be addressed to Z.L. (email: zliu@ frostburg.edu) or F.R. (email: ranfen@163.com)
} 
(i) Open Access This article is licensed under a Creative Commons Attribution 4.0 International License, which permits use, sharing, adaptation, distribution and reproduction in any medium or format, as long as you give appropriate credit to the original author(s) and the source, provide a link to the Creative Commons license, and indicate if changes were made. The images or other third party material in this article are included in the article's Creative Commons license, unless indicated otherwise in a credit line to the material. If material is not included in the article's Creative Commons license and your intended use is not permitted by statutory regulation or exceeds the permitted use, you will need to obtain permission directly from the copyright holder. To view a copy of this license, visit http://creativecommons.org/licenses/by/4.0/.

(C) The Author(s) 2018 